\title{
INVESTIGATION OF UNSTEADY SHEET/CLOUD CAVITATION IN THE DIVERGENT SECTION OF A NOZZLE WITH EMPHASIS ON THE MECHANISM OF SHOCK WAVE PROPAGATION
}

\author{
XIAOBO QUAN ${ }^{1}$, SHAOHUA CHENG ${ }^{1}, \mathrm{CHANGCHANG} \mathrm{WANG}^{2}$, GUOYU WANG $^{2}$ \& BIAO HUANG ${ }^{2}$ \\ ${ }^{1}$ Beijing Institute of Astronautics System Engineering, China \\ ${ }^{2}$ School of Mechanism and Vehicular Engineering, Beijing Institute of Technology, China
}

\begin{abstract}
The objective of this paper is to investigate the unsteady pressure fluctuation characteristics in the process of breakup, and shedding of unsteady sheet/cloud cavitating flows via combined experimental and computational methods. Experiments are conducted in the divergent section of a convergentdivergent channel using a simultaneous sampling technique to synchronize the transient cavitation behaviours and wall-pressure signals. In the numerical simulations, the Zwart cavitation model and the modified RNG $k-\varepsilon$ turbulence model are solved, with the compressibility effects of both water and vapour considered. In addition, one-dimensional bubbly shock wave relationship is applied to analyse the process of the discontinuity propagation. Two different types of cavity breakup and shedding existing in the unsteady sheet/cloud cavitating flows are observed, which is induced by re-entrant flow and discontinuity propagation, respectively. The re-entrant flow generates at the rear of the cavity, moving forward along the wall. When it arrives at the throat, it breaks up the attached cavity, resulting in the cloud cavity shedding. During the process, the wall-pressure fluctuation is relatively small. The discontinuity propagation results from the bubbly shock in water/vapour mixture of the sheet/cloud cavity. There is a significant difference in vapour fraction across the discontinuity. The pre-discontinuity area is almost pure vapour, and the post-discontinuity area consists of water/vapour mixtures with relatively low vapour fraction. During the discontinuity propagation, the pressure peak exists at shock wave front. When the discontinuity arrives at the throat, the void fraction will suddenly decrease, which indicates the low vapour generation rate. Under the convection of the main flow, the attached cavity will be separated from the newly generated vapour, resulting in the attached cavity breaking up and being shed.
\end{abstract}

Keywords: cavitation, cavity breakup and shedding, re-entrant flow, shock wave propagation, pressure peak.

\section{INTRODUCTION}

Cavitation is an abrupt phase change phenomenon that occurs in liquids when the local static pressure drops below the saturated vapour pressure, in a variety of fluid machinery including turbines, pumps and marine propellers [1]-[3]. Occurrence of unsteady cavitation, especially the periodic breakup and shedding of sheet/cloud cavity, can lead to problems such as pressure fluctuations, sudden changes in loads, vibration, noise and erosion [4]-[8].

The experimental studies have identified the presence of two main mechanisms of unsteady cavitation dynamics, namely re-entrant flow mechanism and shock wave propagation mechanism, and most researchers focus on the re-entrant flow mechanism [9]-[14]. The experiments conducted by Stutz and Reboud [15] and Coutier-Delgosha et al. [16] using X-ray attenuation method indicated that the void fraction within the cavity can be up to 0.85 , verifying that the cavity is not very dense under certain conditions. According to the discussion of variation of speed of sound with void fraction in Brennen [17], it should be noted that the speed of sound within the cavity could experience sharp decrease, resulting in high local Mach number. On the other hand, the collapse of cavitation structure, such as 
large-scale bubble clusters, is supposed to release pressure pulses with large magnitude. Due to the sharp decrease in the speed of sound within the attached cavity, the propagation of pressure pulses is associated with another time scale in the flow physics, which could have a great effect on the cavitation dynamics. This indicates the possibility of the existence of shock wave propagation mechanism of cavitation instabilities. Reisman et al. [18], Arndt et al. [19], Leroux et al. [20], and Ganesh et al. [21], have discussed the role of bubbly shock wave in the breakup and shedding of cloud cavity. Although the re-entrant flow mechanism and shock wave propagation mechanism of sheet/cloud cavitation are identified in the experiments, more detailed experiments are still needed to investigate the difference of the interaction between the synchronized cavity developments, and the corresponding pressure fluctuations under the two breakup and shedding mechanisms of sheet/cloud cavitation.

In the present work, we investigate the cloud cavity shedding mechanism in a convergentdivergent channel via synchronizing measurement. The compressible URANS, combining the Tait equation of state for pure water and Peng-Robinson equation for pure vapour is applied to capture more detailed cavitation behaviours. The objectives of this paper are to:

1. Investigate the unsteady pressure fluctuation characteristics in the process of breakup and shedding of sheet/cloud cavitation under the re-entrant flow mechanism and the shock wave propagation mechanism.

2. Identify the properties of the shock wave propagation during the cavitation.

\section{EXPERIMENT SETUP}

Experimental studies are carried out in a closed-loop cavitation tunnel discussed in Huang et al. [22]. The test section is $190 \mathrm{~mm} \times 70 \mathrm{~mm}$ in cross section and $700 \mathrm{~mm}$ in length. In the present study, experiments were conducted in a convergent-divergent channel as shown in Fig. 1(a). The simultaneous sampling system [23] which synchronizes the 4 pressure transducers and high-speed camera by a controller, are shown in Fig. 1(b). Depending on the focus of the investigation, $1024 \mathrm{kHz}$ is used for the pressure transducers and $3000 \mathrm{~Hz}$ was used for the high-speed camera.

The Reynolds number and cavitation number are defined in eqns (1) and (2).

$$
\begin{gathered}
R e=U_{t} H / v, \\
\sigma=\left(p_{\infty}-p_{v}\right) /\left(0.5 \rho_{l} U_{t}^{2}\right),
\end{gathered}
$$

where $U_{t}, H, v, p_{\infty}, p_{v}$ and $\rho_{l}$ are the average velocity at the throat of convergent-divergent test section, the throat height, the water kinematic viscosity, the static pressure, the saturated vapour pressure and the water density, respectively.

The experiments were performed under cloud cavitation conditions, and the average throat velocity $U_{t}=10.2 \mathrm{~m} / \mathrm{s}$ and $U_{t}=11.9 \mathrm{~m} / \mathrm{s}$ were chosen in this study, corresponding to a Reynolds number based on the throat height $R e=0.97 \times 10^{6}$ and $R e=1.10 \times 10^{6}$. The cavitation number $\sigma=0.74$ and $\sigma=0.81$.

The image processing is illuminated in Fig. 2. The mean value of the grayscale along $y$ axis at pixel location $x$ is given in eqn (3).

$$
\bar{g}(x)=\frac{1}{N} \sum_{n=1}^{N} g(x, y)
$$



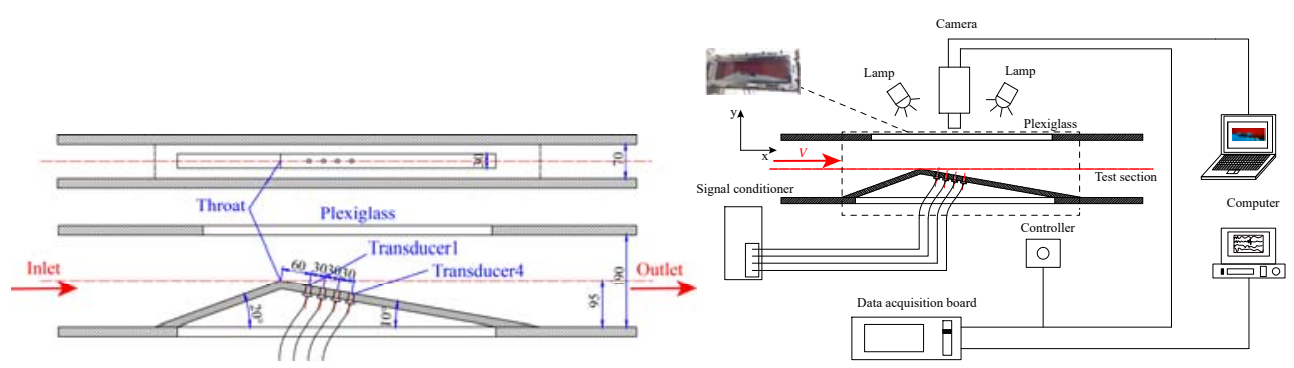

Figure 1: (a) Schematic of the test model; and (b) The simultaneous measurement system.

The variance of the grayscale of along $y$ axis at pixel location $x$ is defined in eqn (4).

$$
\hat{g}(x)=\frac{1}{N} \sum_{n=1}^{N}(g(x, y)-\bar{g}(x))^{2},
$$

where $N$ is the number of pixels along the $y$ axis, $g(x, y)$ is the gray level at pixel $(x, y)$.

\section{NUMERICAL MODEL}

The equation of state of each phase is incorporated into the governing equations to consider the compressibility effects in cavity region. The Tait equation of state [24] for the pure liquid phase is expressed in eqn (5).

$$
\rho_{l}=\left(p+p_{c}\right) /\left(R_{l}\left(T+T_{c}\right)\right)
$$

The Peng-Robinson equation of state [25] for the pure vapour phase is given in eqn (6).

$$
p_{v}=R T_{v} /\left(1 / \rho_{v}-b\right)-a(T) /\left(\left(1 / \rho_{v}\right)^{2}+2 b / \rho_{v}-b^{2}\right)
$$

The Zwart cavitation model [26] and the standard RNG $k-\varepsilon$ model [27] with the modified turbulent viscosity [28] defined as $\mu_{t}=C_{\mu} \rho_{m} k^{2} / \varepsilon f(\rho)$, where $f(\rho)=\rho_{v}+\left(1-\alpha_{v}\right)^{n}\left(\rho_{l}-\rho_{v}\right)$, were used to predict the cavity breakup and shedding dynamics in the present work. This modification has been validated for cavitating flows around convergent-divergent sections [29] and hydrofoils [30], [31].
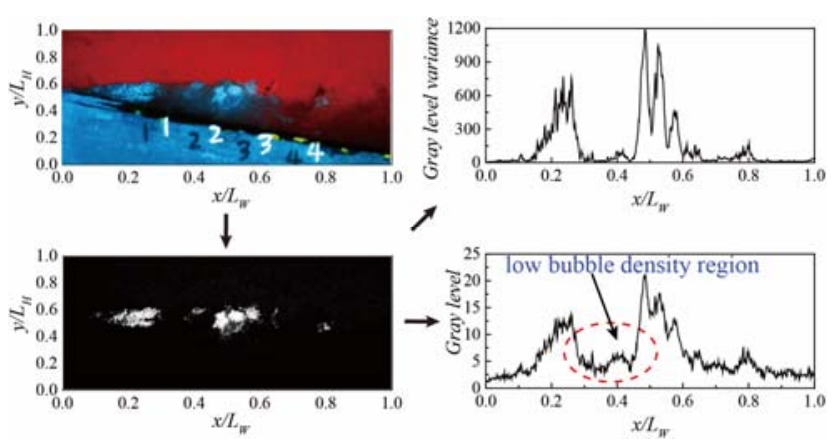

Figure 2: Typical flow visualization and schematic interpretation in experiment (left), and the averaged gray level profile and gray level variance at on instance (LW-the width of the image, LH-the height of the image). 


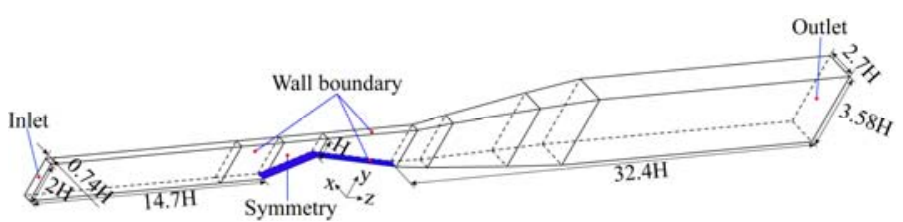

(a)

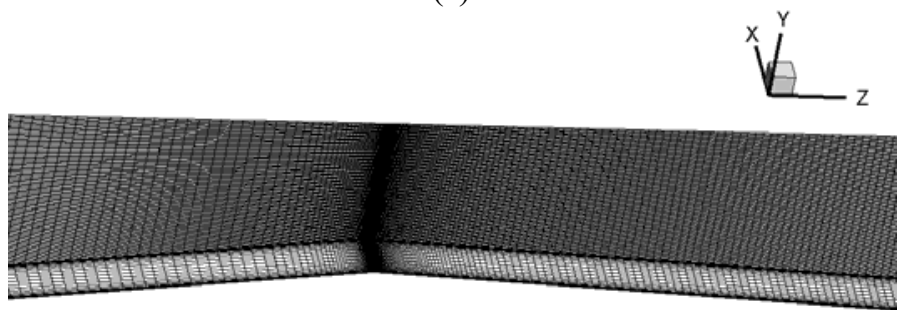

(b)

Figure 3: (a) Computation domain and imposed boundary conditions; and (b) The 3-D magnified view of the convergent-divergent channel.

The computational domain follows the boundaries of the cavitation tunnel, as shown in Fig. 3(a). A special contraction of the mesh is applied in the main flow direction just after the throat to better simulate the two-phase flow area shown in Fig. 3(b).

\section{RESULTS AND DISCUSSION}

\subsection{Global multiphase structures associated with sheet/cloud cavitating flows}

Fig. 4 gives the comparisons of the experimentally observed cavitation pattern with the average gray level distribution and gray level variance along the horizontal direction (left) and predicted vapour fraction iso-surface contours (right, $\alpha_{v}=0.1$ ) under the condition of re-entrant flow induced cavity breakup and shedding for $U_{t}=11.9 \mathrm{~m} / \mathrm{s}, \sigma=0.81, R e=1.10 \times 10^{6}$, and the pressure wave propagation induced cavity breakup and shedding for $U_{t}=10.2 \mathrm{~m} / \mathrm{s}$, $\sigma=0.74, R e=0.97 \times 10^{6}$. Fig. 5 gives the averaged gray level distribution and gray level variance distribution map during three cycles. As shown in Fig. 4, it can be found that the quasi-periodic sheet/cloud cavitation evolution for both the re-entrant flow and shock wave propagation mechanism can be divided into three stages: (1) growth of the attached cavity, (2) the development of the re-entrant flow (the propagation of the shock wave), (3) breakup and shedding and collapse of the attached cavity. It should be noted that during the propagation of shock wave, there exists sharp decrease in void fraction at the shock front, while during the development of re-entrant flow, no evident void fraction change is found. The sharp decrease in void fraction within the cavity will significantly change the cavity dynamics through changing the local speed of sound, and thus the local Mach number [21]. The propagation speed of the shock wave within the attached cavity based on both the high-speed video and the averaged gray level contour is estimated to be $7.12 \mathrm{~m} / \mathrm{s}$.

4.2 Unsteady pressure fluctuation characteristics in the process of breakup and shedding of sheet/cloud cavitation

The unsteady pressure coefficient is defined in equation 7.

$$
C_{p}=\frac{p_{i}}{0.5 \rho U_{t}^{2}}
$$

where, $p_{i}$ is the unsteady fluctuation pressure. 
Re-entrant flow mechanism

Exp.

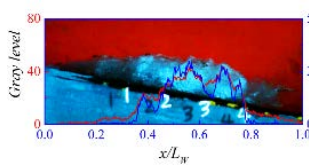

(a) $t_{0}$

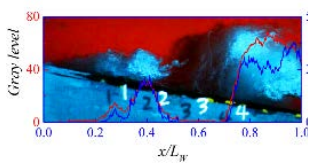

(b) $t_{0}+0.36 T_{\text {ref-re }}$

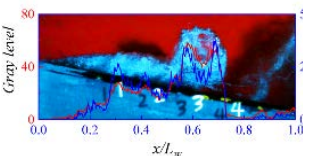

(c) $t_{0}+0.52 T_{\text {ref-re }}$

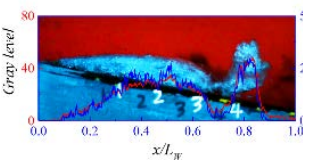

(d) $t_{0}+0.68 T_{\text {ref-re }}$

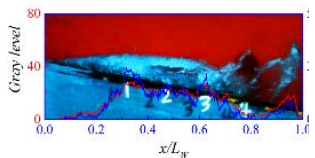

(e) $t_{0}+0.78 T_{\text {ref-re }}$

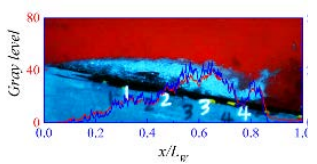

(f) $t_{0}+0.88 T_{\text {ref-re }}$

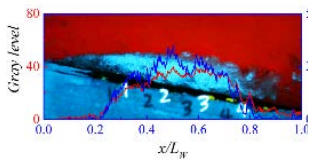

(g) $t_{0}+0.97 T_{\text {ref-re }}$

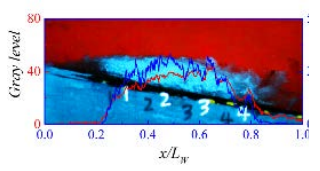

(h) $t_{0}+T_{\text {ref-re }}$
Shock wave propagation mechanism Exp.

Num.
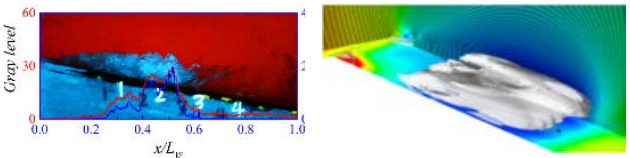

(a) $t_{0}$
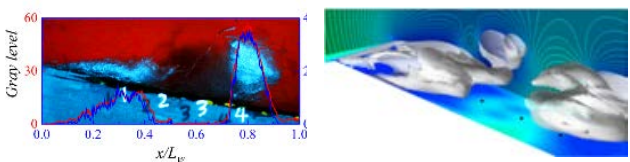

(b) $t_{0}+0.53 T_{\text {ref-sh }}$
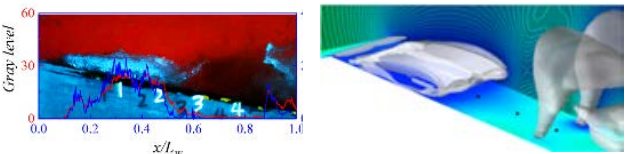

(c) $t_{0}+0.67 T_{\text {ref-sh }}$
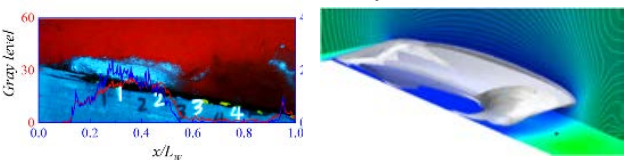

(d) $t_{0}+0.70 T_{\text {ref-sh }}$
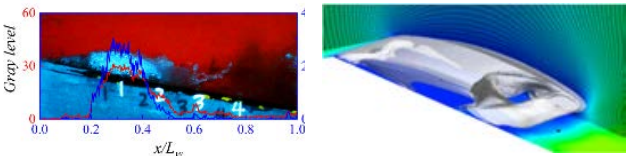

(e) $t_{0}+0.79 T_{\text {ref-sh }}$
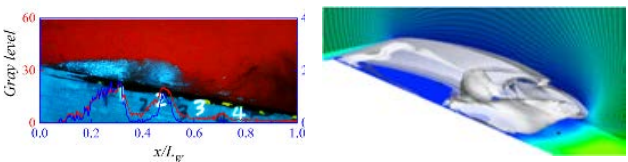

(f) $t_{0}+0.87 T_{\text {ref-sh }}$
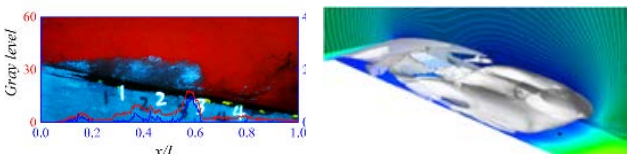

(g) $t_{0}+0.95 T_{\text {ref-sh }}$
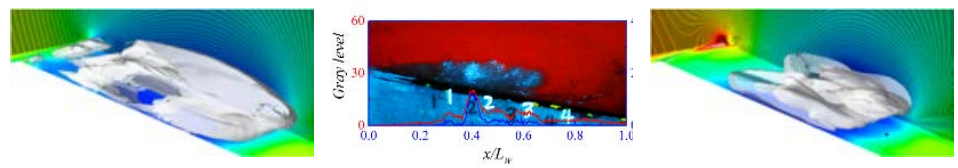

(h) $t_{0+} T_{r e f-s h}$

$0.00 \mathrm{e}+000 \quad 2.50 \mathrm{e}+004 \quad 5.00 \mathrm{e}+004 \quad 7.5 \mathrm{e}+004 \quad 1.00 \mathrm{e}+005$

Figure 4: Comparisons of the experimentally observed cavitation pattern (left), gray level distribution and gray level variance along the horizontal direction and predicted vapour fraction iso-surface contours (right, $\alpha_{v}=0.1$ ) for $U_{t}=11.9 \mathrm{~m} / \mathrm{s}, \sigma=0.81$, $R e=1.10 \times 10^{6}$ and for $U_{t}=10.2 \mathrm{~m} / \mathrm{s}, \sigma=0.74, R e=0.97 \times 10^{6}$. 

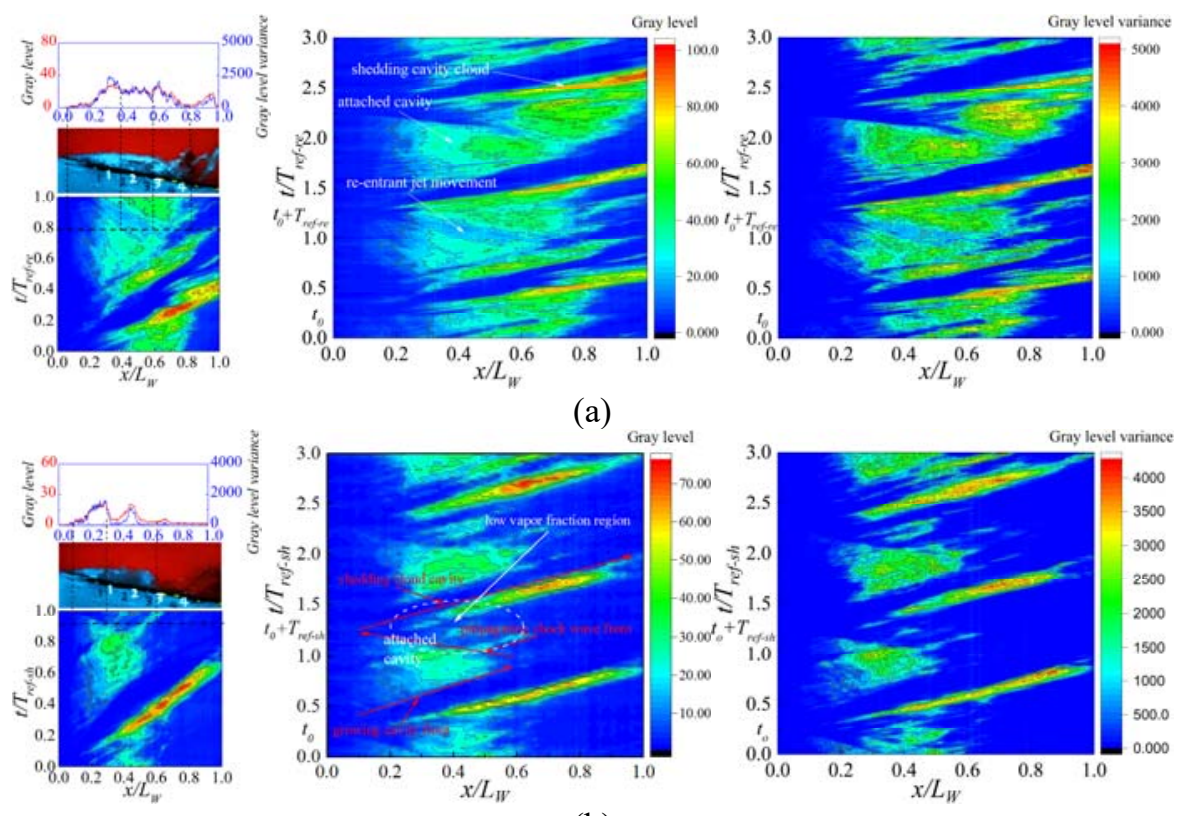

(a)

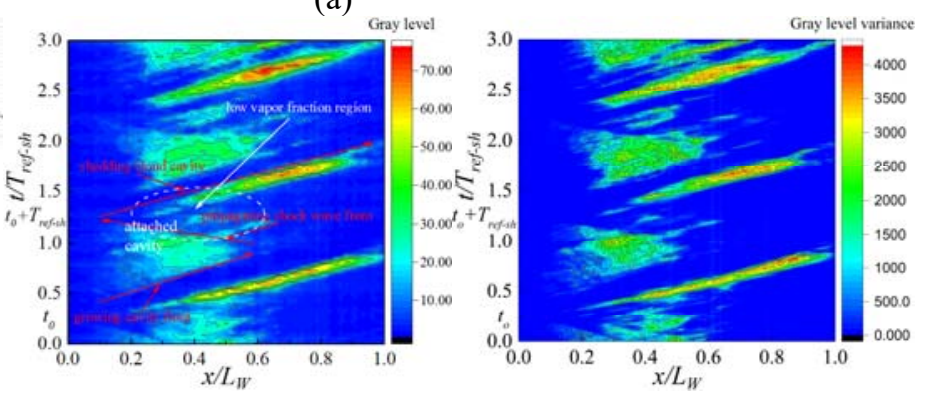

(b)

Figure 5: A typical gray level profile with the corresponding shape of the cavity and the gray level profile and the gray level variance at $t=t_{0}+0.78 T_{\text {ref-re }}$ for (a) and at $t=t_{0}+0.87 T_{\text {ref-sh }}$ for (b) (left), the time evolution of the averaged gray level profile during almost three cycles (middle), and the time evolution of the gray level variance profile during almost three cycles (right) for $U_{t}=11.9 \mathrm{~m} / \mathrm{s}, \sigma=0.81$, $R e=1.10 \times 10^{6}$ and for $U_{t}=10.2 \mathrm{~m} / \mathrm{s}, \sigma=0.74, R e=0.97 \times 10^{6}$.

The corresponding unsteady pressure fluctuations for both re-entrant flow for (a) and shock wave propagation for (b) induced cavity breakup and shedding mechanism during three cycles on four transducers 1, 2, 3 and 4 measured simultaneously with the cavitation images as shown in Fig. 4 are presented in Fig. 6. The images shown in Fig. 4 and the three cavitating flow stages are marked. The red line in Fig. 6 is the trend line plotted by every 800 pressure samples. Combined with the synchronous observations of the experiment images in Fig. 4, it can be seen in Fig. 6 that during the growth of the attached cavity, four transducers, one after another, captures the change of pressure fluctuation when the cavity grows to the position of the transducer. When the transducers are covered by the cavity, relatively steady pressure fluctuations are captured, indicating the steady state within the attached cavity. Compared with the small pressure fluctuation during the development of the re-entrant flow, peak pressure fluctuation as indicated by the red arrows in Fig. 6(b) is captured during the shock wave propagation, which evidently change the local void fraction at the shock wave front within the attached cavity.

To further investigate the pressure fluctuation characteristics during the shock wave propagation, the pressure fluctuation coefficient evolution between $t=t_{0}+0.75 T_{\text {ref-sh }}$ to $t=t_{0}+0.90 T_{\text {ref-sh }}$ as indicated by the red wire frame in Fig. 6 is presented in Fig. 7(a), and the corresponding power spectral density of the pressure fluctuations is presented in Fig. 7(b) to analyse the correlation between the pressure pulses on transducer 1 and transducer 2 . As 


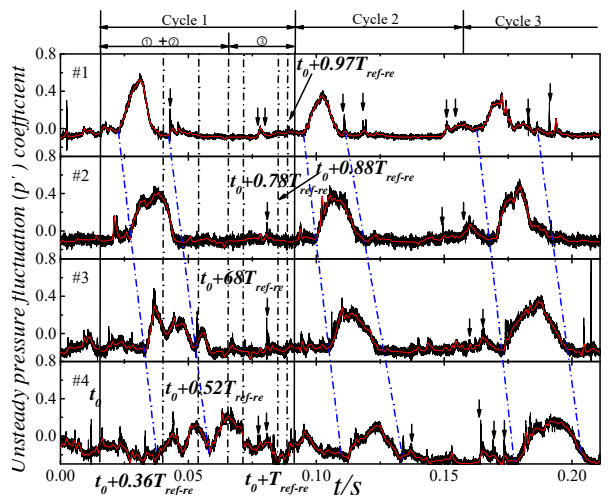

(a)

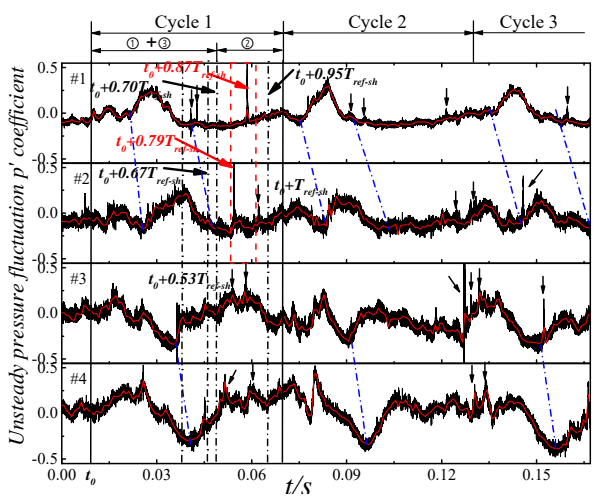

(b)

Figure 6: Experimental unsteady pressure fluctuation coefficient over approximately three cycles for (a) $U_{t}=11.9 \mathrm{~m} / \mathrm{s}, \sigma=0.81, R e=1.10 \times 10^{6}$ and (b) $U_{t}=10.2 \mathrm{~m} / \mathrm{s}, \sigma=0.74$, $R e=0.97 \times 10^{6}$.
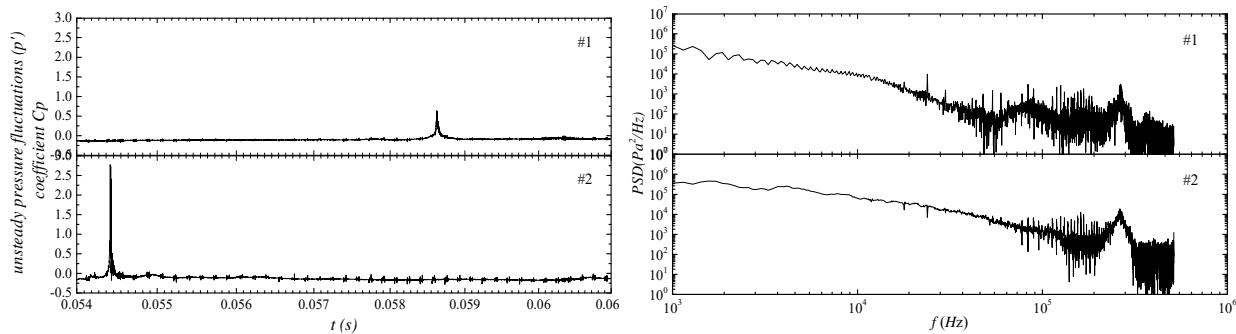

Figure 7: (a) Pressure fluctuation coefficient during the propagation of the shock wave on transducer 1 and 2; and (b) Power spectral density of the corresponding pressure fluctuations on transducer 1 and 2.

can be seen, a peak exists at about $260 \mathrm{kHz}$ on both the two transducers, which is believed to be caused by the same shock wave propagation event. The difference in magnitudes of the pressure pulse detected by the two transducers is supposed to be due to the energy dissipation in the shock wave propagation process.

In the present work, the pressure cross-correlation based on the pressure signal in Fig. 7(a) is also done to investigate the pressure pulses on transducer 1 and transducer 2 as shown in Fig. 8. The cross-correlation is defined in eqn (8).

$$
\mathrm{C}(\mathrm{x}, \mathrm{y}, \tau)=\frac{E\left[p_{1}(x, t) p_{2}(y, t+\tau)\right]}{\sqrt{p_{1}^{2}(x, t) p_{2}^{2}(y, t+\tau)}}
$$

From Fig. 8, it can be found that the pressure signals on transducer 1 and transducer 2 are closely related. The high correlation appears on $-0.0042 \mathrm{~s}$ which is just the time delay between transducer 1 and transducer 2 . Based on the time delay, the propagation speed of the shock wave is estimated to be $7.14 \mathrm{~m} / \mathrm{s}$, which is consistent with that obtained by the high-speed video. 


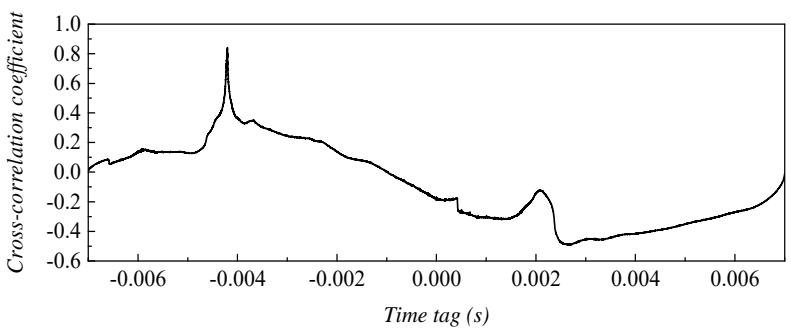

Figure 8: Correlation between the pressure fluctuations on transducer \#1 and transducer \#2 during the shock wave propagation.

To confirm the characteristics of the shock wave, the one-dimension bubbly shock wave relationship is applied. Ignoring the bubble dynamics of the shocking process, the speed upstream of the discontinuity in the reference frame of the shock is obtained in eqn (9) [21].

$$
u_{1}^{2}=\frac{p_{2}-p_{1}}{\rho_{L}}\left[\frac{\left(1-\alpha_{1}\right)}{\left(1-\alpha_{2}\right)\left(\alpha_{1}-\alpha_{2}\right)}\right]
$$

where, 1) the parameter after the shock wave and 2) the parameter before the shock wave, $u_{1}$ is the propagation speed of shock wave towards the throat, $\alpha$ is the void fraction.

Fig. 9 presents the typical instances of the predicted unsteady water vapour fraction contour and the absolute pressure contour (left) and velocity vector distribution (right) during the propagation of the discontinuity. 50 samples points used to evaluate the shock wave propagation speed are evenly distributed along the red line in Fig. 9. It should be noted that the propagation of the shock wave will significantly influence the local flow physics as shown in Fig. 9. The pressure pre- and post-shockwave have a jump, with higher pressure and higher velocity post of the shock wave. Based on the numerical results of the flow parameters across the shock wave, the averaged shock wave propagation speed using the eight instance is 7.5 $\mathrm{m} / \mathrm{s}$. this value is close to that obtained by visualizations and pressure signal cross-correlation, identifying the discontinuity to be a kind of bubbly shock wave.

\section{CONCLUSIONS AND FUTURE WORK}

Unsteady pressure fluctuations accompanying with the cavitation behaviours under the two different types of breakup and shedding mechanisms of unsteady sheet/cloud cavitating flows are analysed. The primary findings are the following:

- The experimental and numerical results demonstrate that, there exist two different breakup and shedding mechanisms in the quasi-periodic sheet/cloud cavitating flows, namely re-entrant flow induced sheet/cloud cavitation shedding mechanism and shock wave propagation induced sheet/cloud shedding mechanism.

- For the re-entrant flow induced sheet/cloud cavitation shedding mechanism, the re-entrant flow generates at the rear of the attached cavity and moves upstream along the wall beneath the attached cavity. During the propagation of the re-entrant flow, the pressure fluctuations are relatively small, due to the relatively steady state of the water/vapour mixture within the attached cavity.

- For the shock wave propagation induced sheet/cloud cavitation shedding mechanism, the shock wave is produced by the collapse of large scale cavity 
structure, such as cloud cavity, and propagates within the attached cavity. During the propagation of the shock wave, pressure peak exists at the shock wave front. Much higher-pressure fluctuations are captured by pressure transducers and a significant void fraction difference can be observed across shock wave front. The physical parameters pre- and post-shockwave front satisfy the one-dimensional shock wave relationship which proves the void fraction discontinuity to be induced by a bubbly shock wave.

Regarding future work, additional research through combined experimental investigation and numerical modelling methods are needed to further advance the understanding of the transient between the two breakup and shedding mechanism. More research is also needed to improve and validate the compressible multiphase turbulence closure model and the mass transfer model to better capture the cavitation structure associated with the highly compressibility effects of the cavitation region. Considering that cloud cavitation will cause

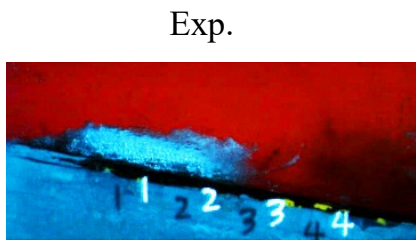

Vapor/absolute pressure

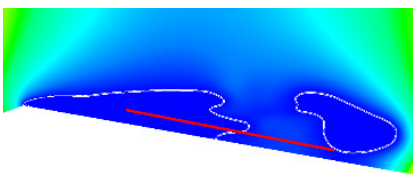

(a) $t_{0}+0.70 T_{\text {ref-sh }}$
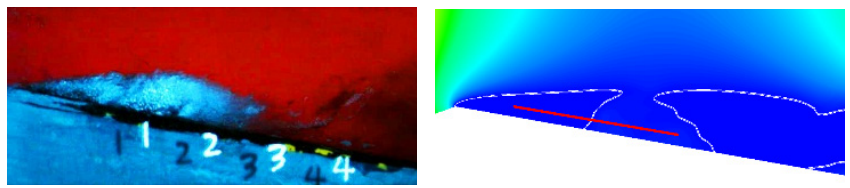

(c) $t_{0}+0.80 T_{\text {ref-sh }}$
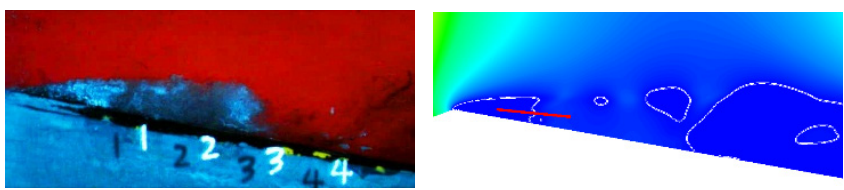

(e) $t_{0}+0.90 T_{\text {ref-sh }}$
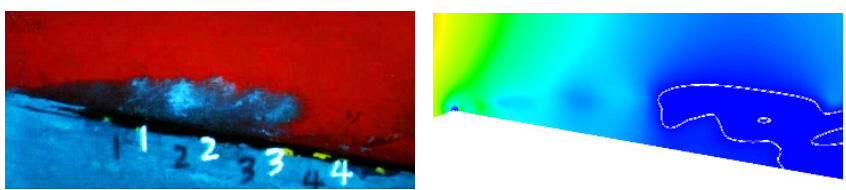

(h) $t_{0}+T_{\text {ref-sh }}$
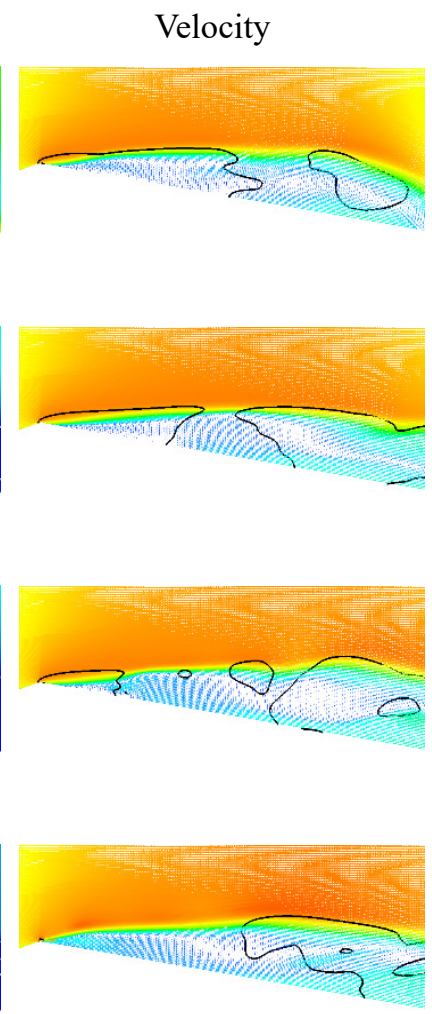

Velocity $\mathrm{W}[\mathrm{m} / \mathrm{s}]$

Absolute pressure [Pa]

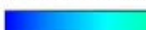

$\begin{array}{llllllllll}3.0 \mathrm{e}+003 & 9.8 \mathrm{e}+003 & 1.7 \mathrm{e}+004 & 2.3 \mathrm{e}+004 & 3.0 \mathrm{e}+004 & -3.0 \mathrm{e}+000 & 1.3 \mathrm{e}+000 & 5.5 \mathrm{e}+000 & 9.8 \mathrm{e}+000 & 1.4 \mathrm{e}+001\end{array}$

Figure 9: Comparisons of the predicted unsteady water vapour fraction contour and the absolute pressure contour (left) and velocity distribution (right) during the shock wave propagation. 
serious pressure fluctuations, vibration, noise and so on, additional experiments and numerical studies are needed to investigate the effects of air-ejection on the depress of pressure fluctuations induced by the re-entrant flow mechanism and shock wave propagation mechanism, respectively.

\section{ACKNOWLEDGEMENTS}

The authors gratefully acknowledge support by the National Science Foundation of China (NSFC, Grant Nos: 51239005 and 51679005), National Natural Science Foundation of Beijing (Grant No: 3172029) and the Open Foundation of State Key Laboratory of Ocean Engineering (Shanghai Jiao Tong University, China).

\section{REFERENCES}

[1] Knapp, R.T., Daily, J.W. \& Hammitt, F.G., Cavitation, McGraw Hill: New York, 1970.

[2] Brennen, C.E., Cavitation and Bubble Dynamics, Oxford University Press: New York, 1995.

[3] Wang, G., Senocak, I., Wei, S., Ikohagi, T. \& Cao, S., Dynamics of attached turbulent cavitating flows. Progress in Aerospace Sciences, 37(6), pp. 551-581, 2001.

[4] Liang, J., Luo, X., Liu, Y., Li, X. \& Shi, T., A numerical investigation in effects of inlet pressure fluctuations on the flow and cavitation characteristics inside water hydraulic poppet valves. International Journal of Heat \& Mass Transfer, 103, pp. 684700, 2016.

[5] Chen, G., Wang, G., Hu, C., Huang, B. \& Zhang, M., Observations and measurements on unsteady cavitating flows using a simultaneous sampling approach. Experiments in Fluids, 56(2), pp. 1-11, 2015.

[6] Joseph, D.D., Cavitation in a flowing liquid. Physical Review E Statistical Physics Plasmas Fluids \& Related Interdisciplinary Topics, 51(3), pp. R1649, 1995.

[7] Paik, B.G., Kim, K.S., Kim, K.Y., Ahn, J.W. \& Kim, T.G., Test method of cavitation erosion for marine coatings with low hardness. Ocean Engineering, 38(13), pp. 1495$1502,2011$.

[8] Wu, Q., Huang, B., Wang, G. \& Gao, Y., Experimental and numerical investigation of hydroelastic response of a flexible hydrofoil in cavitating flow. International Journal of Multiphase Flow, 74, pp. 19-33, 2015.

[9] Knapp, R.T., Recent investigations of the mechanics of cavitations and cavitation damage. Trans Asme, 77, 1955.

[10] Furness, R.A. \& Hutton, S.P., Experimental and theoretical studies of two-dimensional fixed-type cavities. Journal of Fluids Engineering, 97(4), pp. 515-521, 1975.

[11] Kawanami, Y., Kato, H., Yamaguchi, H., Tanimura, M. \& Tagaya, Y., Mechanism and control of cloud cavitation. Journal of Fluids Engineering, 119(4), pp. 788-794, 1997.

[12] Stutz, B. \& Reboud, J.L., Experiments on unsteady cavitation. Experiments in Fluids, 22(3), pp. 191-198, 1997.

[13] Stutz, B. \& Reboud, J.L., Two-phase flow structure of sheet cavitation. Physics of Fluids, 9(12), pp. 3678-3686, 1997.

[14] Callenaere, M., Franc, J.P., Michel, J.M. \& Riondet, M., The cavitation instability induced by the development of a re-entrant jet. Journal of Fluid Mechanics, 444, pp. 223-256, 2001.

[15] Stutz, B. \& Reboud, J.L., Measurements within unsteady cavitation. Experiments in Fluids, 29(6), pp. 545-552, 2000. 
[16] Coutier-Delgosha, O., Stutz, B., Vabre, A. \& Legoupil, S., Analysis of cavitating flow structure by experimental and numerical investigations. Journal of Fluid Mechanics, 578, pp. 171-222, 2007.

[17] Brennen, C.E., Fundamentals of Multiphase Flow, Cambridge University Press: England, 2005.

[18] Reisman, G.E., Wang, Y. \& Brennen, C.E., Observations of shock waves in cloud cavitation. Journal of Fluid Mechanics, 355, pp. 255-283, 1998.

[19] Arndt, R.E.A., Song, C.C.S., Kjeldsen, M., He, J. \& Keller, A., Instability of partial cavitation: A numerical/experimental approach. Symposium on Naval Hydrodynamics, 2001.

[20] Leroux, J.B., Coutier-Delgosha, O. \& Astolfi, J.A., A joint experimental and numerical study of mechanisms associated to instability of partial cavitation on two-dimensional hydrofoil. Physics of Fluids, 17(5), pp. 515-513, 2005.

[21] Ganesh, H., Mäkiharju, S.A. \& Ceccio, S.L., Bubbly shock propagation as a mechanism for sheet/cloud transition of partial cavities. Journal of Fluid Mechanics, 802, pp. 37-78, 2016.

[22] Huang, B., Yin, L.Y., Wang, G.Y. \& Wei, S., Combined experimental and numerical computational investigation of unsteady structure of sheet/cloud cavitation. Journal of Fluids Engineering, 135(7), pp. 71-301, 2013.

[23] Chen, G., Wang, G., Hu, C., Huang, B., Gao, Y. \& Zhang, M., Combined experimental and computational investigation of cavitation evolution and excited pressure fluctuation in a convergent-divergent channel. International Journal of Multiphase Flow, 72, pp. 133-140, 2015.

[24] Chen, H.T. \& Collins, R., Shock wave propagation past an ocean surface. Journal of Computational Physics, 7(7), pp. 89-101, 1971.

[25] Peng, D.Y. \& Robinson, D.B., Industrial \& Engineering Chemistry Fundamentals, American Chemical Society, 15, pp. 59-64, 1976.

[26] Zwart, P.J., Gerber, A.G. \& Belamri, T., A two-phase flow model for predicting cavitation dynamics. Proceedings of the Fifth International Conference on Multiphase Flow, 152, 2004.

[27] Orszag, S.A. et al., Renormalization group modeling and turbulence simulations. International Conference on Near-wall Turbulence Flows, pp. 1031-1046, 1993.

[28] Coutier-Delgosha, O., Reboud, J.L. \& Delannoy, Y., Numerical simulation of the unsteady behaviour of cavitating flows. International Journal for Numerical Methods in Fluids, 42(5), pp. 527-548, 2003.

[29] Deaix, J. \& Goncalvès, E., Compressible effects modeling in turbulent cavitating flows. European Journal of Mechanics-B/Fluids, 39, pp. 11-31, 2013.

[30] Dular, M., Bachert, R., Schaad, C. \& Stoffel, B., Investigation of a re-entrant jet reflection at an inclined cavity closure line. European Journal of Mechanics-B/Fluids, 26(5), pp. 688-705, 2007.

[31] Li, D.Q., Grekula, M. \& Lindell, P., Towards numerical prediction of unsteady sheet cavitation on hydrofoils. Proceedings of the 9th International Conference on Hydrodynamics, pp. 741-746, 2010. 